\title{
PARAMETRIC STUDY ON AIR CORE PHENOMENA AT LIQUID DRAINING FROM CYLINDRICAL TANK
}

\author{
I. S. Park ${ }^{1}$, C. H. Sohn ${ }^{1}$ \\ ${ }^{1}$ School of Mechanical Engineering, Kyungpook National University (corresponding- \\ einstein@knu.ac.kr)
}

\begin{abstract}
An air core phenomenon observed from lavatory bowl draining in our daily lives has a very important scientific meaning because it is also observed from many industrial applications and related with a natural phenomenon such as tornado. Moreover since the air core affects the drain speed, it is deeply connected with a disaster such as flood from local heavy rain. Thus many studies preventing the air core from forming have been conducted and various types of vortex suppressor have been suggested. In this paper, the numerical simulation on air core phenomenon is introduced and a lot of simulating results such as cross-sectional flow field, air-core-formation time and so on are discussed in view of the reason of forming the air core. Simulations on various sized tanks and drain ports, and the initial rotating speed of cylinder wall have been conducted (total 56 cases). The container level heights firstly forming the air core were compared from each other case. From dimensional analyses, four non-dimensional parameters and the relating equation among them were determined.
\end{abstract}

Keywords: Air core, Swirl velocity, Draining.

\section{INTRODUCTION}

The air core can be observed in the various industrial fields such as the molten steel tundish in a continuous-casing process and the sump pump station. The phenomenon is also connected with the sewerage design because the air core could severely decrease the draining flow rate.

Despite considerable research efforts [1-8] in the hydraulic engineering field to elucidate the air core vortexing phenomenon, it still requires the more enhanced theories and experimental bases. In this study, the phenomenon is fully numerically investigated. We test the free draining from the cylindrical tank. The progression of the free surface and the fluid flow inside the tank are numerically visualized at various times.

For preventing or suppressing the generation of the air core, the various attempts have been applied by the previous researchers [9-12]. However, we focused on the observing the physics inside the phenomenon rather than suggesting the new-type vortex suppressors be- 
cause the detailed understand for the phenomenon is more helpful to devise the high performance suppressor.

We varied the tank and drain port diameters as well as the swirl velocities so as to establish the criterion for the air core generation. Through a parametric study for those factors, the influence of each factor was appraised. For these, the time that the dip fully penetrates the liquid inside (the critical time) and the level difference between the initial state and the critical time (the air core generation distance or critical distance) are measured numerically for various cases. Moreover by dividing the critical distance by the critical time, the new concept of the critical speed for air core genernation was develped.

Several non-dimensional parameters such as the diameter ratio for the tank and the drain port, the Reynolds number, the Froude number, the Weber number, and the nondimensional air core generation speed are suggested from the dimensional analysis. A correlation for the dimensionless parameters was completed to determine the vortex core generation for different cases.

\section{NUMERICAL ANALYSIS}

For the transient, incompressible, laminar, and free-surface flows in an axisymmetric assumption, the following conservation equations for mass and momentum were selected as the set of governing equations.

$$
\begin{gathered}
\frac{\partial}{\partial x_{i}}\left(\rho u_{i}\right)=0 \\
\frac{\partial\left(\rho u_{i}\right)}{\partial t}+\frac{\partial}{\partial x_{j}}\left(\rho u_{i} u_{j}\right)=-\frac{\partial p}{\partial x_{j}}+\frac{\partial}{\partial x_{j}}\left[\mu\left(\frac{\partial u_{i}}{\partial x_{j}}+\frac{\partial u_{j}}{\partial x_{i}}\right)\right]+\rho g_{i}
\end{gathered}
$$

Here, $x_{i}$ is the position vector of the coordinate system, $u_{i}$ the velocity vector, $t$ the time, $p$ the pressure, $\rho$ the density, and $\mu$ the dynamic viscosity.

The cylindrical tank firstly was rotated for 10 seconds. We replaced the event as rotating the tank side wall with a constant angular velocity. Next the rotating wall was stoped. The free rotating and viscous dissipating process was retained for 3 seconds. Finally the drain port was opened and the draining was started.

The present flow pattern can be assumed to have no circumferential gradient of the flow variables. However the flow obviously has the circumferential component of velocity, which has a spatial distribution in the tank and slowly decays as time goes on. To find the swirl velocity component in a two-dimensional grid system, the following conservation equation for the tangential momentum was applied in addition to the basic axisymmetric governing equation set, Eq. (1) and (2).

$$
\frac{\partial}{\partial z}\left(r \rho u_{z} u_{\theta}\right)+\frac{\partial}{\partial r}\left(r \rho u_{r} u_{\theta}\right)=\frac{\partial}{\partial z}\left(r \mu \frac{\partial u_{\theta}}{\partial z}\right)+\frac{1}{r} \frac{\partial}{\partial r}\left(r^{3} \mu \frac{\partial}{\partial r}\left(\frac{u_{\theta}}{r}\right)\right)-\rho u_{r} u_{\theta}
$$

Here, $z$ is the axial component of the coordinate system, $r$ the radial coordinate component, $u_{r}$ the radial velocity component, and $u_{\theta}$ the tangential velocity component. Eq. 

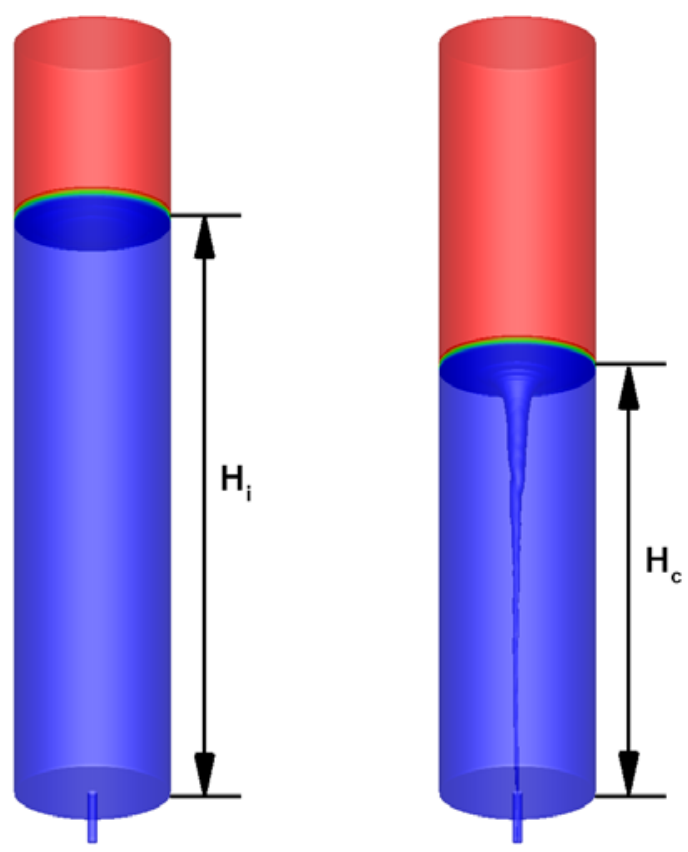

Figure 1. Initial level and critical height.

(3) is the conservation equation of the circumferential momentum in a cylindrical coordinate system by eliminating all circumferential gradient terms. This is very useful and practical in terms of the computational cost because the high level flow information, including all three velocity components can be obtained on a two-dimensional mesh system.

To capture the shape of air core, the VOF (Volume Of Fluid) scheme [13] was adopted, To update the free surface shape progression, while the basic flow fields are being calculated implicitly, the implicitly free surface updating and HRIC convective scheme for the volume fraction equation are applied for minimum computational costs.

The FVM (Finite Volume Method) based commercial CFD code, Fluent 6.3, was used. For the boundary condition at the opened top of the tank and the drain port exit, the constant pressure condition of $1 \mathrm{~atm}$. was applied. The no-slip condition was applied for all walls. The surface tension coefficient between water and air was kept constant at $0.0712 \mathrm{~N} / \mathrm{m}$ and the gravitational acceleration was constant a $9.81 \mathrm{~m} / \mathrm{s}^{2}$. The time step size was set as $1 \times 10^{-3} \sim 1 \times 10^{-4}$ seconds.

A schematic of the system is shown in Figure 1. The reference cylinder tank had a 90 mm diameter, and a $450 \mathrm{~mm}$ height. The reference diameter of the drain port was $6 \mathrm{~mm}$ and its height was $15 \mathrm{~mm}$. The tank was initially filled up with water to a level $\left(H_{i}\right)$ of $350 \mathrm{~mm}$ from the bottom of the tank. And its top was opened so that the liquid water interfaces with ambient air of $1 \mathrm{~atm}$. For the numerical simulations, 390 nodes were allocated in the axial direction and a total of 45 nodes were used in the radial direction. Of course the number of nodes used in simulations varied case-by-case according to the dimensions of the corresponding cases. However, the intervals between the neighboring nodes were usually kept within 1-2 $\mathrm{mm}$ in all cases. 


\section{RESULTS AND DISCUSSIONS}

Firstly we checked the drain time for the full draining. The cases that the side wall initially rotates or not, were compared with each other. The total drain time had a big difference, more than 20 seconds between the cases. The declining pattern of the water level was displayed in Figure 2. A peculiar thing is that the initially rotating case shows a deflection of the water level near at 20 second. On the other hand, the non-rotating case shows a monotonic decrease of the water level. In the rotating case, the air core is generated at around 20 second. The air core generation is closely connected with the draining flow rate because the effective discharging area severly decrease if the vortex core generates. However, the decreasing slops of the water level after 35 second are almost same for both the rotating or non-rotating cases. Once the air core is generated, the core is usually maintained till the drainging is finished. That means although the vortex core exists, the draining flow rate is getting recovered as the draining continues.

Figure 2 also presents the check of grid dependency. A total 25 and 45 grids for radial direction and 200 and 390 grids for axial direction are tested for the grid dependency check. All grid systems well simulate the deflection at around 20 second. Moreover the descending slops of the water level after the vortex core generation are predicted similarly for all cases. However the times for the recovery of the drain flow rate are slightly different. The coarser grid system shows the longer recovery time. Anyway we used the 45 grids for radial direction and 390 grids for axial direction in this study.

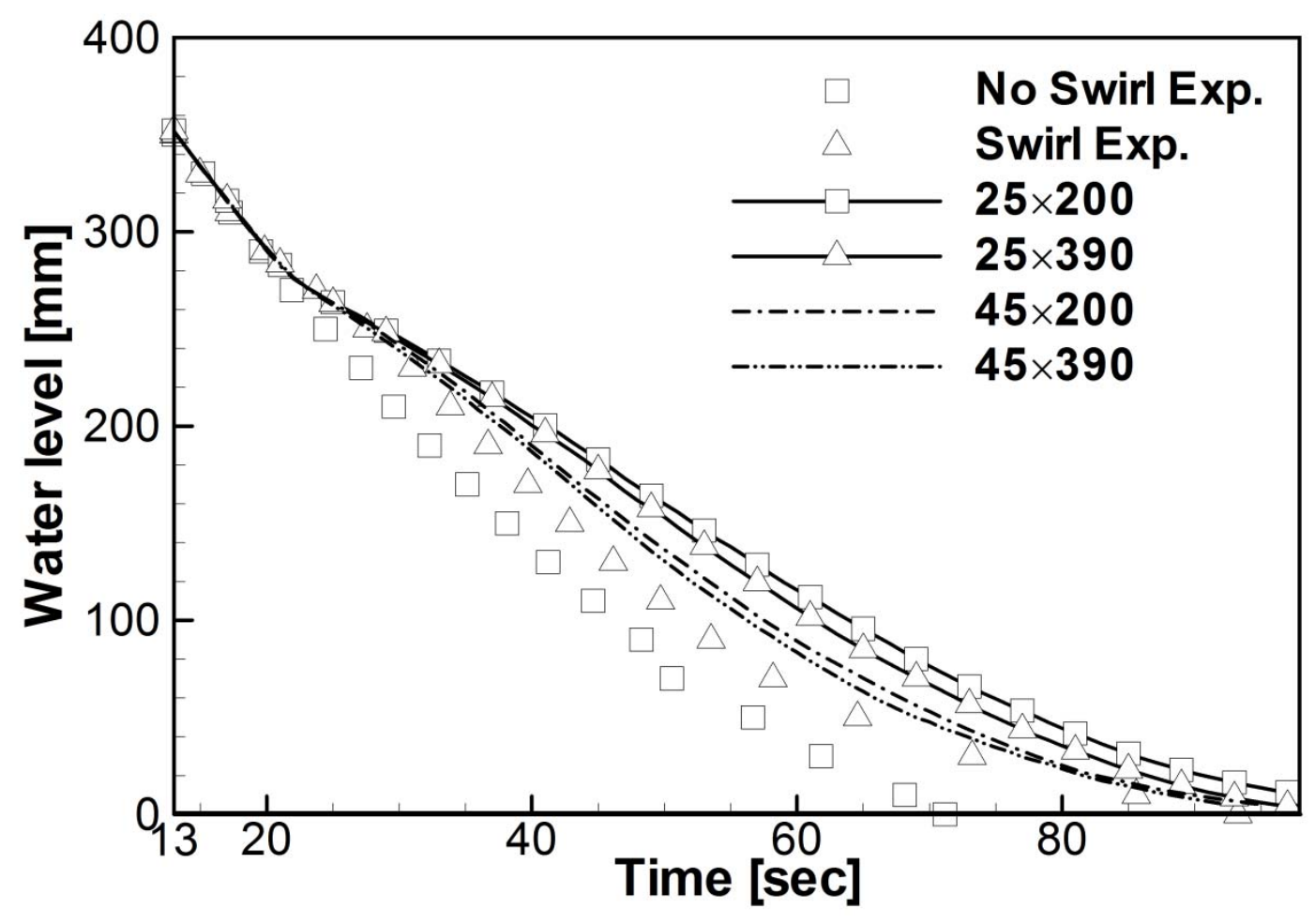

Figure 2. Water level declining history. 

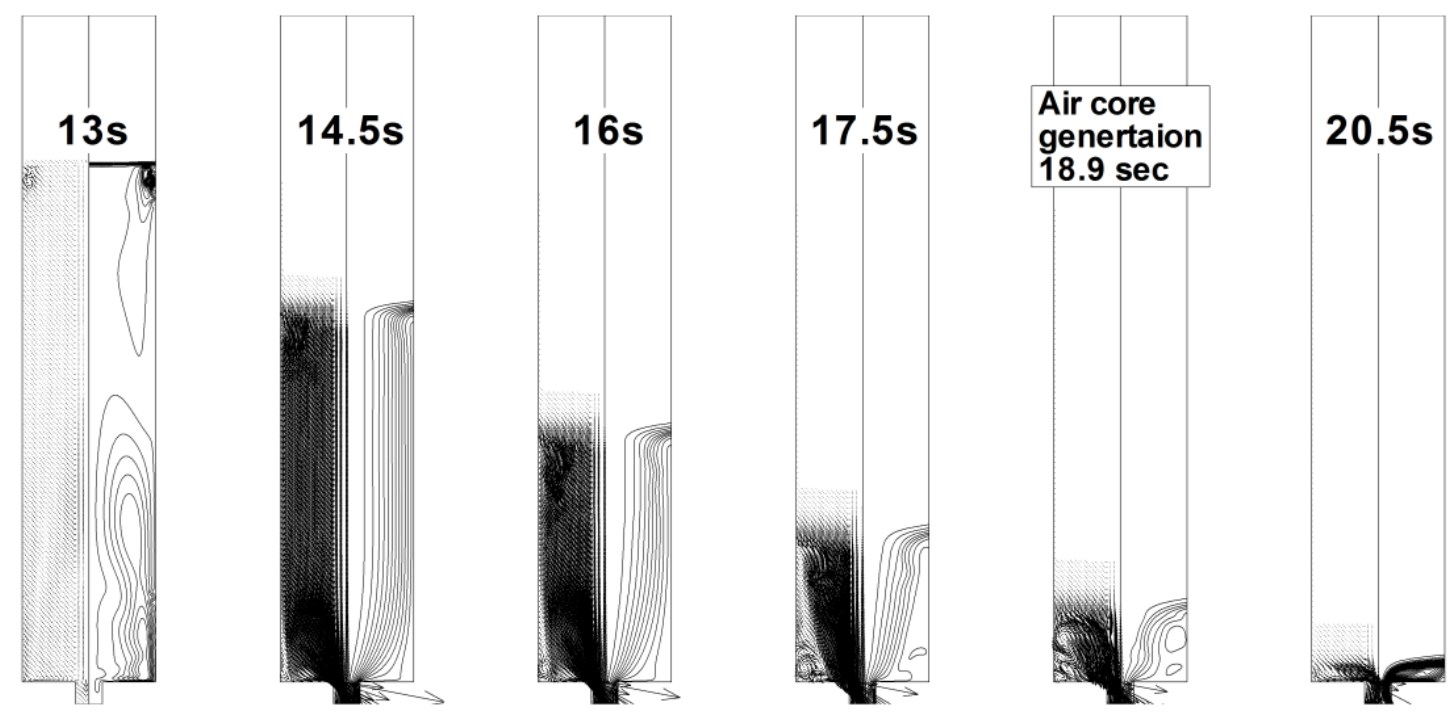

(a) Initial tank rotation $40 \mathrm{rpm}$
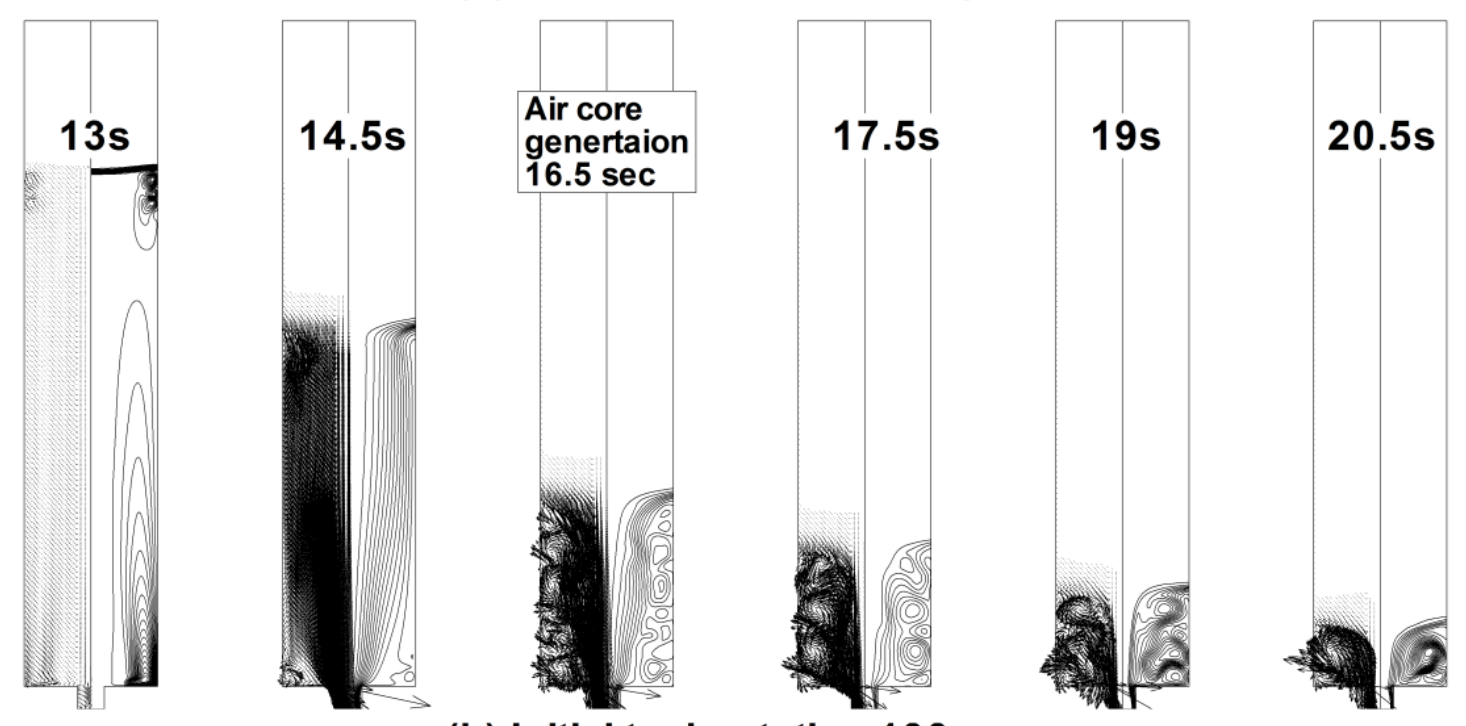

(b) Initial tank rotation $100 \mathrm{rpm}$
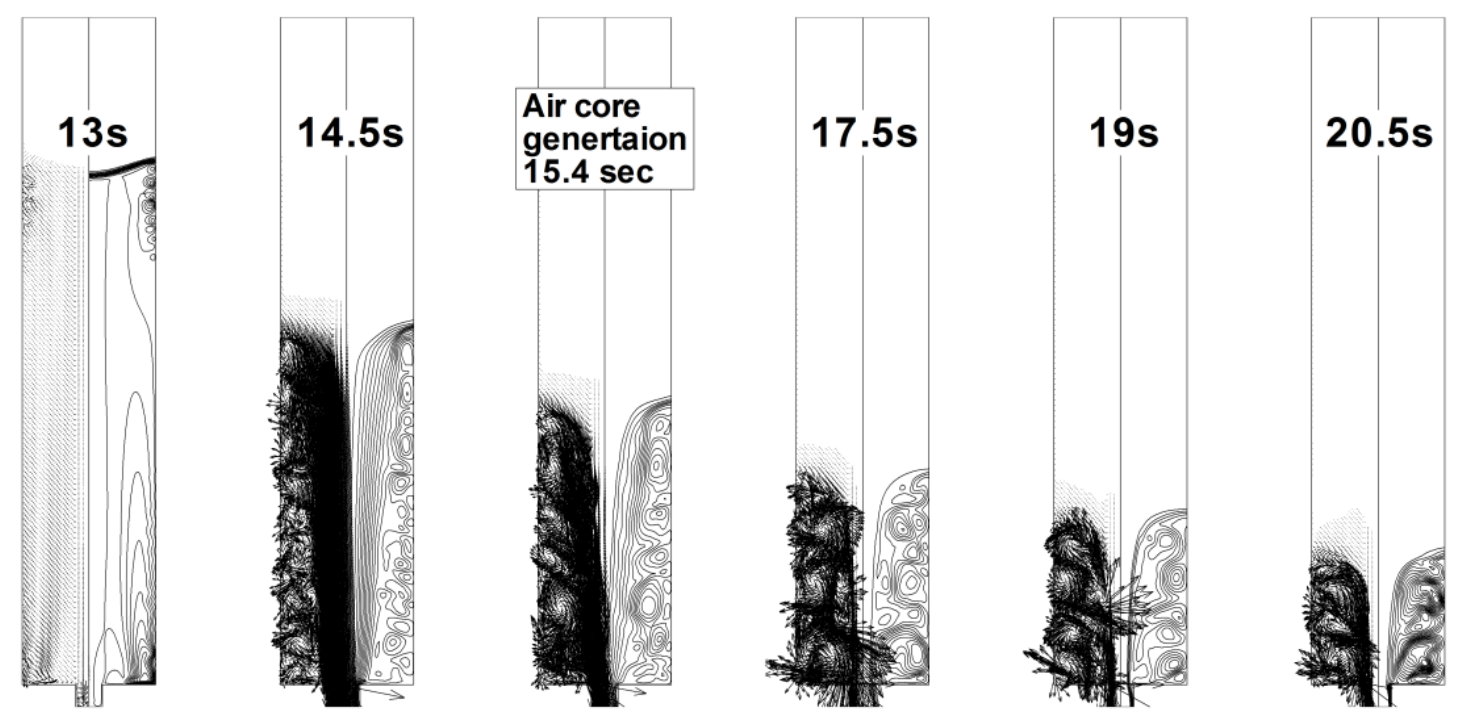

(c) Initial tank rotation $180 \mathrm{rpm}$

Figure 3. Velocity vectors and stream lines for various tank roation speeds. 
In Figure 3 the time progression of the internal flow structures for three different initial swirl speeds are presented. To exclude the influences of other parameters, the tank diameter is kept at $90 \mathrm{~mm}$ and the drain-port diameter at $18 \mathrm{~mm}$. The results for the initial swirl speeds of 40,100, and $180 \mathrm{rpm}$ are compared together. As the swirl speed increases, the Taylor vortex on the side wall is generated earlier. On the other hand, the case with lower swirl speed shows the Taylor vortex generation at a later time. The flow pattern before the Taylor vortex are structured inside the tank looks like the well stratified free drain and the streamlines parallel to the axis. The air core generation strongly relates to not only the Taylor vortex formation but also the its growth. The torus-shape vortex confines the large parts of the liquid inside the tank and concentrates the liquid drain into the centered region.

We simulated a total 56 cases of $D=90$ and $120 \mathrm{~mm}, d=6,12,18$, and $24 \mathrm{~mm}$, and the tank rotation speed $=40,60,80,100,120,150$, and $180 \mathrm{rpm}$. We've check the time and the water level when the air core is generated for the first time. Next for the generalization, we made some non-dimensional variables, i.e., the diameter ratio of tank to drain port $D^{*}=D / d$, the rotational Reynolds number $R e=\rho D^{2} \omega / \mu$, the rotational Froude number $F r=D \omega^{2} / g$, the rotational Weber number $W e=\rho D^{3} \omega^{2} / \sigma$, and the non-dimensional critical speed $V^{*}=V_{c} / D \omega$. Here, $D$ is the tank diameter, $d$ the drain port diameter, $\rho$ the density, $\omega$ the tank roation speed in rad/sec., $\mu$ the dynamic viscosity, $g$ the gravitational acceleration, $\sigma$ the surface tension coefficient, and $V_{c}$ the critical velocity. The critical velocity is defined by the following;

$$
V_{c}=\frac{H_{i}-H_{c}}{t_{c}}
$$

here, $H_{i}$ is the initial water level, $H_{c}$ the critical height (i.e., the water level when the air core is generated), $t_{c}$ the critical time (i.e., the time when the air core is generated).

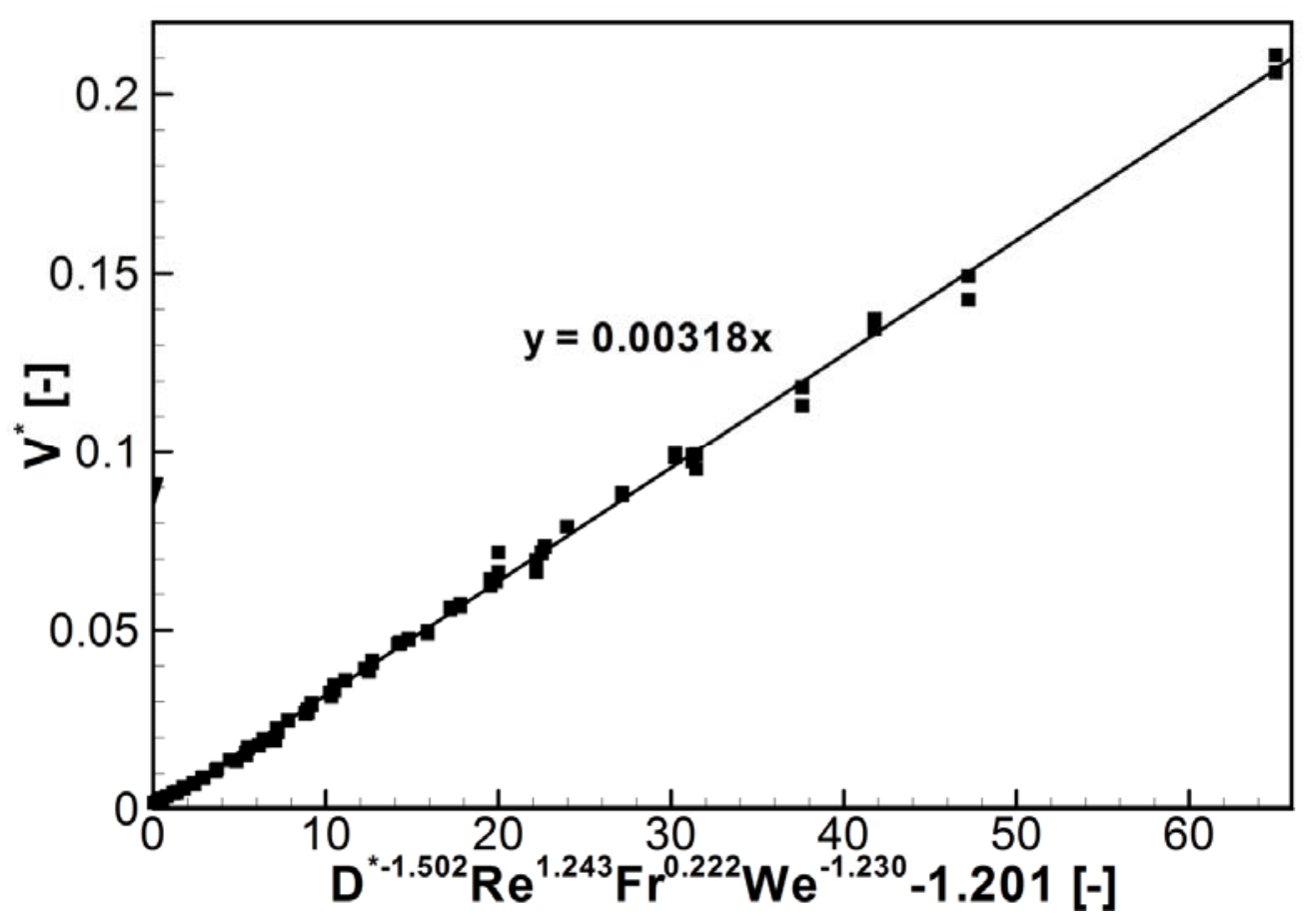

Figure 4. Correlation among non-dimensional parameters. 
From the numerical results, we made the following correlation among the nondimensional parameters.

$$
V^{*}=0.00318\left(D^{*-1.502} \operatorname{Re}^{1.243} \operatorname{Fr}^{0.222} W e^{-1.230}-1.201\right)
$$

\section{CONCLUSIONS}

The vortexing core phenomenon is fully numerically simulated. The FVM based CFD skills and the VOF free surface tracking method are applied. The drain time or the water level descending speed are checked to prove the consistency of the numerical results. Some characteristic features are well reproduced by the present CFD study. Expanding the calculating range for the geometrical dimension and the tank rotation speed, we made the correlation equation to explain or determine the air core generation.

\section{Acknowledgements}

This research was supported by Basic Science Research Program through the National Research Foundation of Korea (NRF) funded by the Ministry of Education, Science and Technology (the Grant 2010-0024619).

\section{REFERENCES}

[1] H. O. Anwar, “Formation of a weak vortex”. Journal of Hydraulic Research. 4, 1-16, 1966

[2] H. O. Anwar, J. A. Weller, and M. B. Amphlett, "Similarity of free-vortex at horizontal intake”. Journal of Hydraulic Research. 16, 95-105, 1978.

[3] L. L. Daggett, and G. H. Keulegan, "Similitude in free-surface vortex formations". Journal of Hydraulic Division-ASCE. 100, 1565-1581, 1974.

[4] R. Granger, “Steady three-dimensional vortex flow”. Journal of Fluid Mechanics. 25, 557-576, 1966.

[5] G. E. Hecker, "Model-prototype comparison of free surface vortices”. Journal of Hydraulic Division-ASCE. 7, 1243-1259, 1981.

[6] A. K. Jain, K. G. R. Raju, and R. J. Garde, "Vortex formation at vertical pipe intakes". Journal of Hydraulic Division-ASCE. 104, 1429-1445, 1978.

[7] A. W. Marris, "Theory of the bathtub vortex". Journal of Applied Mechanics, Transactions of the ASME. 34, 11-15, 1967.

[8] M. Padmanabhan, and G. E. Hecker, "Scale effects in pump sump models”. Journal of Hydraulic Engineering-ASCE. 110, 1540-1556, 1984.

[9] B. H. L. Gowda, P. J. Joshy, and S. Swarnamani, "Device to suppress vortexing during draining from cylindrical tanks”. Journal of Spacecraft and Rockets (AIAA). 33, 598-600, 1996.

[10] B. H. L. Gowda, and H. Udhayakumar, "Vane-type suppressor to prevent vortexing during draining from cylindrical tanks”. Journal of Spacecraft and Rockets (AIAA). 43, 
381-383, 2005.

[11] C. H. Sohn, B. H. L. Gowda, and M. G. Ju, "Eccentric drain port to prevent vortexing during draining from cylindrical tanks". Journal of Spacecraft and Rockets. 45, 638-640, 2008.

[12] K. Ramamurthi and T. J. Tharakan, "Shaped discharge ports for draining liquids". Journal of Spacecraft and Rockets (AIAA). 30, 786-788, 1992.

[13] C. W. Hirt, and B. D. Nichols, "Volume of fluid(VOF) method for the dynamics of free boundaries”. Journal of Computational Physics. 39, 201-225, 1981. 